\title{
Data de Desmame e Desempenho Reprodutivo de Vacas de Corte ${ }^{1}$
}

\section{Luciane Salgueiro Pio de Almeida ${ }^{2}$, José Fernando Piva Lobato ${ }^{3}$, Flávio Schramm Schenkel ${ }^{4}$}

RESUMO - O experimento foi conduzido na Estação Experimental Agronômica da Universidade Federal do Rio Grande do Sul, município de Eldorado do Sul, Brasil. O objetivo do estudo foi avaliar o efeito da data do desmame precoce (05/01/99) ou do convencional (26/03/99) sobre o desempenho reprodutivo de vacas de corte multíparas em duas estações de monta, 98/99, e a subseqüente, 99/00. Cinqüenta vacas tiveram seus bezerros desmamados em média aos 91 dias de vida, com peso mínimo de $70 \mathrm{~kg}($ desmame precoce $=$ DP $)$, e 43 vacas desmamaram em média aos 170 dias, com peso médio de 131,2 kg (desmame convencional = DC). Os períodos de acasalamento ocorreram de 01/12/98 a 26/02/99, de 06/12/99 a 28/02/00. As vacas do DP apresentaram ganho médio diário (GMD) superior e melhor condição corporal (CC) que as do DC no primeiro acasalamento $(0,828 \mathrm{~kg}$ x 0,549 kg; 2,4 x 2,0; P<0,05). O índice de prenhez em 1999 foi de $40,0 \%$ x $11,1 \%$ e em 2000 de 51,4\% x 20,0\%, para o DP e DC, respectivamente. Não foi observada diferença no intervalo entre partos. A taxa de prenhez no primeiro acasalamento foi menor que o esperado, devido possivelmente à baixa CC no início do acasalamento e à baixa recuperação durante o mesmo. Mesmo assim, o DP propiciou melhor CC às vacas durante o primeiro acasalamento, possibilitando índices reprodutivos significativamente melhores aos do desmame convencional.

Palavras-chave: condição corporal, desempenho reprodutivo, idade de desmame, taxa de prenhez, vacas de corte

\section{Weaning Data and the Reproductive Performance of Beef Cows}

\begin{abstract}
The experiment was run at the Agronomic Experimental Station of the Federal University of Rio Grande do Sul, located in the district of Eldorado do Sul, Brazil. The objective of the study was to evaluate the effect of the date of early (01/05/99) or conventional $(03 / 26 / 99)$ weaning on the reproductive performance of multiparous beef cows during two mating seasons, $98 / 99$, and the subsequent, 99/00. Fifty cows had their calves weaned with an average age of 91 days old and a minimum liveweight of $70 \mathrm{~kg}($ early weaning $=\mathrm{EW})$, and 43 cows had calves weaned with an average age of 170 days old and mean liveweight of $131.2 \mathrm{~kg}$ (conventional weaning $=\mathrm{CW})$. The mating periods were from $12 / 01 / 98$ to $02 / 26 / 99$ and from $12 / 06 / 99$ to $02 / 28 / 00$. Cows of EW had higher daily liveweight gains and better body condition $(\mathrm{BC})$ than $\mathrm{CW}$ during the first breeding season $(0.828 \mathrm{~kg}$ x $0.549 \mathrm{~kg} ; 2.4 \times 2.0 ; \mathrm{P}<0.05)$. The pregnancy rate in 1999 was $40.0 \%$ x $11.1 \%$ and in 2000 it was $51.4 \%$ x $20.0 \%$ for EW and CW, respectively. There was no difference in the calving intervals. The pregnancy rate at first mating was lower than the expected, probably due to the low $\mathrm{BC}$ at beginning of the mating season and to the low recovery during the mating period. The EW improved cows $\mathrm{BC}$ during the first mating, making possible significantly better reproductive rates than the $\mathrm{CW}$.
\end{abstract}

Key Words: beef cows, body condition, pregnancy rate, reproductive performance, weaning age

\section{Introdução}

Os custos crescentes da pecuária de corte e a importância econômica do incremento dos índices reprodutivos incentivam o desenvolvimento e a utilização de práticas de manejo como o desmame precoce, o qual melhora a eficiência dos sistemas de produção pelo aumento da porcentagem de prenheze diminuição do intervalo parto-concepção (Pimentel et al., 1979).

Wiltbank (1970) afirma ser a pequena produção de bezerros e o longo período da estação reprodutiva os dois principais indicadores de problemas reprodutivos em rebanhos de cria.

A freqüência, a intensidade e a duração da amamentação têm sido consideradas como os determinantes primários da duração do anestro no pós-parto (Williams, 1990).

O nível de nutrientes digestíveis totais (NDT) no pré e pós-parto afeta a performance reprodutiva de vacas de corte adultas (Wiltbank et al., 1962). As pesquisas mostram estar o nível nutricional no pósparto condicionado pela magnitude do nível nutricional

\footnotetext{
${ }^{1}$ Parte da tese na Faculdade de Agronomia - UFRGS do $1^{\circ}$ autor como um dos requisitos para obtenção do título de Mestre em Zootecnia, Área de Concentração Produção e Manejo Animal.

2 Médica Veterinária, M.Sc. em Zootecnia - UFRGS, Porto Alegre, RS. E.mail: milaemar@ig.com.br

3 Professor Adjunto IV, Ph.D., Departamento de Zootecnia, Faculdade de Agronomia - UFRGS, Caixa Postal 776, Porto Alegre, RS, CEP: 90001-970. Bolsista do CNPq. E.mail: lobato@orion.ufrgs.br

4 Pesquisador Associado da Universidade de Guelph, ON, Canada. E.mail: schenkel@uoghelph.ca
} 
pré-parto. Quando o nível no pré-parto é muito baixo e prolongado, havendo forte queda de peso vivo, o nível no pós-parto é significativamente mais importante em determinar o momento do aparecimento do primeiro cio no pós-parto. Entretanto, quando as vacas parem em bom estado corporal, o nível alimentar no pós-parto tem menor efeito sobre o comportamento reprodutivo (Rovira, 1996).

Lobato et al. (1998b), trabalhando com vacas mestiças primíparas (Tabapuã ou Nelore x Devon), verificaram que o nível alimentar alto no pré-parto associado ao nível alimentar alto no pós-parto aumenta o índice de prenhez e proporciona intervalos de partos (IEP) inferiores a 365 dias.

Lobato et al. (2000), trabalhando com vacas primíparas aos três anos de idade mestiças zebuínas e taurinas, com desmama precoce (DP) ou convencional (DC) aos seis meses de idade, observaram menores IEP (359,61 dias vs 381,63 dias) para vacas submetidas a DP, porém as taxas de prenhez foram semelhantes $(100 \%$ vs $89,47 \%),(P>0,05)$. O DP também concentrou a segunda parição no início da estação de partos, dando mais tempo às vacas de conceber do parto ao final da terceira estação de monta. Além disso, vacas concebendo tarde na estação de monta produzem bezerros menores ao desmame (Quadros \& Lobato, 1996).

Osoro \& Wright (1992) verificaram que vacas Hereford x Holandês e Blue-Greys, parindo com altos escores de CC diminuíram seus IEP em 11,2 dias a cada unidade de $\mathrm{CC}$ ao parto (escala de $0=$ magra; $5=$ gorda). Estes autores relataram que a CC no início do acasalamento apresentou menor importância para o IEP, sendo que a CC ao final da estação de monta não apresentou efeito. Neste trabalho, apenas $42 \%$ da variação do IEP foi explicada pelos fatores estudados.

O objetivo deste trabalho foi estudar os efeitos de duas datas de desmame, precoce e convencional, sobre o desempenho reprodutivo de vacas de corte multíparas mantidas em campo nativo, analisando o índice de prenhez, o IEP e a CC no ano do desmame. Na temporada de monta seguinte foram medidos os efeitos das datas de desmame prévias sobre o índice de prenhez.

\section{Material e Métodos}

O experimento foi conduzido na Estação Experimental Agronômica da Universidade Federal do Rio Grande do Sul, situada no Km 146 da BR 290, município de Eldorado do Sul, pertencente à região da
Depressão Central do Estado do Rio Grande do Sul, no período de janeiro de 1999 a janeiro de 2001.

O clima da região é do tipo $\mathrm{Cfa}$ (subtropical úmido), segundo a classificação de Köppen (Bergamaschi \& Guadagnin, 1990). A precipitação média anual é de 1400 mm, com menor incidência nos meses de verão. O solo pertence à unidade de mapeamento São Jerônimo, classificado como laterítico bruno avermelhado distrófico (Brasil, 1973). Possui textura franco argilosa à argilosa, sendo solos ácidos, pobres em matéria orgânica, com pH em torno de 5,0.

A pastagem nativa é composta por espécies dos gêneros Andropogon, Axonopus e Paspalum, com leguminosas como Trifolium polimorphum e Desmodium incanum. Há gramíneas cespitosas de baixo valor forrageiro como Erianthus angustifolis (macega estaladeira) e invasoras como Eryngium horridum (caraguatá), Baccharis trimera (carqueja) e Baccharis coridifolia (mio-mio) (Boldrini, 1993).

Foram utilizadas 93 vacas multíparas mestiças (Bos taurus x Bos indicus) com cria ao pé com graus de sangue entre $1 / 4$ a $3 / 4$ zebu e com três a nove anos de idade. Os bezerros nasceram no período de 01 de setembro a 14 de novembro de 1998 . O período de cobrição foi de 01/12/98 a 26/02/99, com 3\% de touros testados, sendo o DP realizado em 05/01/99. A segunda estação de cobrição foi realizada de 06/12/99 a $28 / 02 / 00$. As vacas foram manejadas em campo nativo durante todo o experimento.

As estimativas da disponibilidade de forragem foram realizadas a cada 28 dias pelo método comparativo (Haydock \& Shaw, 1975). Foram realizados cinco cortes de $0,25 \mathrm{~m}^{2}$ rentes ao solo, sendo pontuados de um (menor disponibilidade forrageira) a cinco (máxima disponibilidade forrageira). Foram feitas 30 avaliações visuais aleatórias e pontuadas de um a cinco de acordo com os cortes previamente realizados no potreiro. As amostras foram acondicionadas em sacos de papel identificados de acordo com o potreiro e o dia da coleta. No Laboratório de Nutrição Animal da Faculdade de Agronomia da Universidade Federal do Rio Grande do Sul, foram pesadas (matéria verde) e mantidas em estufa $\left(60^{\circ} \mathrm{C}\right)$ por 72 horas, sendo pesadas novamente para determinação da matéria seca. Após, o material foi moído. O teor de nitrogênio foi determinado pelo método de Kjeldahl, sendo multiplicado por 6,25 para ser obtido o teor de PB da amostra (A.O.A.C., 1975).

Os tratamentos experimentais foram:

- Desmame precoce (DP): 50 vacas com 
bezerros(as) submetidos ao desmame precoce com mínimo de $70 \mathrm{~kg}$ de peso vivo em $05 / 01 / 99$, com peso vivo médio de $82,3 \mathrm{~kg}$ e idade média de 91 dias.

- Desmame convencional (DC): 43 vacas com bezerros(as) desmamados em 26/03/99, com peso vivo médio de $131,2 \mathrm{~kg}$ e idade média de 170 dias.

Todas as vacas e seus bezerros foram pesados a cada 28 dias a partir de 05/01/99, data do DP, oportunidade em que também foram e passaram a ser classificadas de acordo com suas CC, numa escala de 1 a 5 (Lowman et al., 1973). Os bezerros(as) foram submetidos a jejum por aproximadamente 15 horas antes das pesagens.

A taxa de prenhez das vacas no ano de 1999 foi determinada por palpação retal em 03/05/99 e no ano de 2000, a prenhez foi diagnosticada em 25/05/00.

O IEP das vacas que repetiram prenhez na monta de 01/12/98 a 26/02/99 foi determinado com base nas datas de parição de 1998 e 1999.

Odelineamento experimental utilizado foi o Delineamento Completamente Casualizado (DCC). A análise dos dados foi realizada utilizando o programa estatístico Statistical Analysis System (Sas Institute, 1996).

Os pesos e ganhos de peso $(\mathrm{kg})$ das vacas, com distribuições assumidas normais, e a CC (escores) durante o acasalamento, com distribuição discreta, foram analisadas pelo método dos quadrados mínimos, utilizando o procedimento General Linear Models (GLM), que considera o desbalanceamento do número de observações (Sas Institute, 1996). A variável IEP (dias) foi analisada por análise de sobrevivência, utilizando o procedimento LIFETEST do SAS. O método do produto-limite foi utilizado para a obtenção das estimativas das funções de sobrevivência. Uma vaca que não concebeu na segunda estação de monta foi considerada "right-censored".

O modelo estatístico utilizado foi o seguinte:

$$
\begin{gathered}
\mathrm{Y}_{\mathrm{hijl}}=\mu+\mathrm{D}_{\mathrm{h}}+\mathrm{G}_{\mathrm{i}}+\mathrm{S}_{\mathrm{j}}+\mathrm{DG}_{\mathrm{hi}}+\mathrm{b}_{1}(\mathrm{DJP})+\mathrm{b}_{2} \\
\left(\mathrm{DJP}^{2}\right)+\mathrm{b}_{3} \mathrm{IV}+\mathrm{e}_{\mathrm{hijl}}
\end{gathered}
$$

em que: $\mathrm{Y}_{\text {hijl }}=$ variável resposta associada ao 1-ésimo animal; $\mu=$ média geral; $D_{h}=$ efeito fixo do h-ésimo tipo de desmame; $G_{i}=$ efeito fixo do i-ésimo grupo genético materno; $\mathrm{S}_{\mathrm{j}}=$ efeito fixo do j-ésimo sexo dos bezerros; $\mathrm{DG}_{\mathrm{hi}}$ = efeito fixo da interação entre o hésimo tipo de desmame e o i-ésimo grupo genético materno; IV = idade da vaca; DJP = data juliana do parto (anterior); $b_{1}$ e $b_{3}=$ coeficientes de regressão linear; $b_{2}=$ coeficiente de regressão quadrático; $\mathrm{e}_{\mathrm{hijl}}=$ efeito residual aleatório.

$O$ índice de prenhez das vacas foi analisado pelo teste do Qui-Quadrado (Gomez \& Gomez, 1984), utilizando o procedimento FREQ do SAS com a opção "cmh" para ajustar para outras fontes de variação, que não aquelas da tabela de contingência, utilizando o modelo abaixo:

$$
Y_{h i j l}=\mu+D_{h}+G_{i}+I V_{j}+e_{h i j l}
$$

em que: $\mathrm{Y}_{\mathrm{hijl}}=$ índice de prenhez associado à l-ésima vaca; $\mu=$ média geral; $D_{h}=$ efeito fixo do h-ésimo tipo de desmame; $\mathrm{G}_{\mathrm{i}}$ = efeito fixo do i-ésimo grupo genético materno; $\mathrm{IV}_{\mathrm{j}}=$ efeito fixo da j-ésima idade da vaca; $\mathrm{e}_{\mathrm{hijl}}=$ efeito residual aleatório

\section{Resultados e Discussão}

Na Tabela 1 estão apresentados os pesos vivos e $\mathrm{CC}$ das vacas na data do desmame precoce (PDP e CDP) e ao final do primeiro acasalamento (PFA e CFA), de acordo com os tratamentos.

Os pesos vivos e CC observados no início do acasalamento foram semelhantes, porém são considerados baixos para a obtenção de bons desempenhos reprodutivos.

Uma vaca adulta de tipo e cruzamento semelhante ao deste experimento em bom estado nutricional deve apresentar, no início do acasalamento, entre $380 \mathrm{a}$ $400 \mathrm{~kg}$ para ter bom desempenho reprodutivo, o que equivale a CC de 3,5 a 4,0 pontos (escala de 1 a 5) (Rovira, 1996; Lobato et al., 1998a; 1998b; 2000).

O peso ao final do acasalamento (PFA), em 26/02/99, teve efeito do tipo de desmame. As vacas submetidas ao DP foram $(\mathrm{P}<0,05)$ mais pesadas $(11,1 \mathrm{~kg})$ que as

Tabela 1 - Pesos e escores de condição corporal médios das vacas no desmame precoce (PDP e CDP), no final do primeiro acasalamento (PFA e CFA) e número de animais (N), para os tratamentos, desmame precoce (DP) ou convencional (DC)

Table 1 - Mean liveweights and body condition scores at early weaning (EWW and $E B C$ ), at end of the mating period (MFW and MBC) and number of animals (N), for the treatments of early (EW) or conventional weaning (CW)

\begin{tabular}{lccccc}
\hline $\begin{array}{l}\text { Tratamento } \\
\text { Treatment }\end{array}$ & $\mathrm{N}$ & $\begin{array}{c}\text { PDP, } k g \\
E W W\end{array}$ & $\begin{array}{c}\text { CDP } \\
E B C\end{array}$ & $\begin{array}{c}\text { PFA, } k g \\
M F W\end{array}$ & $\begin{array}{c}\text { CFA } \\
M B C\end{array}$ \\
\hline DP & 50 & $359,1^{\mathrm{a}}$ & $1,6^{\mathrm{a}}$ & $402,1^{\mathrm{a}}$ & $2,4^{\mathrm{a}}$ \\
$E W$ & & & & & \\
$\mathrm{DC}$ & 43 & $362,5^{\mathrm{a}}$ & $1,5^{\mathrm{a}}$ & $391,0^{\mathrm{b}}$ & $2,0^{\mathrm{b}}$ \\
$C W$ & & & & & \\
\hline
\end{tabular}

Médias seguidas da mesma letra, na coluna, não diferem significativamente por t teste $(P>0,05)$.

Means followed by the same letter, in a column, do not significativelly differ by $t$-test $(P>$.05).

R. Bras. Zootec., v.31, n.3, p.1223-1229, 2002 
do tratamento DC, provavelmente devido à redução das exigências de nutrientes anteriormente destinadas à lactação, o que possibilitou maiores ganhos de peso para as vacas (Ferreira, 1993).

Os maiores pesos das vacas do DP ao final do acasalamento estão de acordo com os obtidos por Sá et al. (1997), os quais obtiveram em vacas mestiças, com bezerros desmamados aos 60 dias ou aos 120 dias de idade, pesos ao final do acasalamento de $416,3 \mathrm{~kg}$ e $392,5 \mathrm{~kg}$, respectivamente.

Pesos adequados e CC ao início do acasalamento são determinantes para uma maior velocidade de concepção (Wiltbank et al., 1962; Lobato et al., 1998a; 1998b; 2000). Ao final do acasalamento, os mesmos são indicadores do desempenho reprodutivo final das vacas, pois podem evidenciar melhor nível nutricional e maiores ganhos durante o acasalamento, possibilitando taxas de prenhez mais altas (Wiltbank et al., 1962).

Lobato \& Barcellos (1992) trabalhando com vacas multíparas mestiças base Charolês, observaram em vacas que pastaram em pastagem melhorada, com peso médio no início do acasalamento de $394,0 \mathrm{~kg}$, uma taxa de prenhez de $77,9 \%$, enquanto as mantidas em campo nativo, tendo iniciado o acasalamento com média de $372,0 \mathrm{~kg}$, tiveram apenas $27,0 \%$ de prenhez.

Os baixos escores de CC das vacas ao início do primeiro acasalamento foi devido à baixa disponibilidade de matéria seca (MS) existente no campo nativo (730,5 $\mathrm{kg}$ de $\mathrm{MS} / \mathrm{ha})$ e à qualidade protéica $(6,5 \%$ PB) desta forragem. A melhoria de $0,4 \mathrm{em} \mathrm{CC}$ das vacas do DP foi significativa $(\mathrm{P}<0,01)$, evidenciando a melhoria da CC das vacas do DP durante o tempo restante do acasalamento. Em 05/01/99 continuavam com baixas CC, terminando o acasalamento 52 dias após, ainda com CC abaixo das recomendadas (Rovira, 1996), em que pese a diferença obtida entre os animais dos dois tratamentos.

Simeone \& Lobato (1996) encontraram um aumento da CC de vacas mestiças zebu durante o acasalamento, havendo uma superioridade para as vacas que tiveram seus bezerros desmamados no $35^{\circ}$ dia (45 dias antes do fim do acasalamento) após o início do acasalamento (DP) sobre as vacas cujos bezerros foram desmamados em 08/03 (98 dias após o início do acasalamento - DC). Os escores de CC na data do desmame definitivo foram de 3,06 x 2,33 para os grupos DP e DC, respectivamente.

$\mathrm{Na}$ Figura 1 estão os ganhos médios diários (GMD) das vacas da data do DP (05/01/99) ao final do acasalamento (26/02/99).

Os GMD observados foram superiores aos de Lobato \& Barcellos (1992) que, ao desmamarem bezerros de vacas multíparas mestiças base Charolês aos 100 dias (D100) ou aos 180 dias (D180) de idade, obtiveram variação de peso durante o acasalamento de $0,360 \mathrm{~kg} \mathrm{x}-0,022 \mathrm{~kg}$ para D100 e D180, respectivamente. Os altos GMD obtidos são explicados pelo aumento na disponibilidade de forragem em fevereiro de 1999, a qual foi de 3047,2 kg de matéria seca/ha.

Na Tabela 2 são apresentados os índices de prenhez do período 1998/1999 em que as vacas foram submetidas aos tratamentos de desmame, e os do período 1999/2000 quando foram medidos os efeitos dos tratamentos no comportamento reprodutivo subseqüente e os IEP.

No diagnóstico de gestação de 1999, sob efeitos imediatos do DP ou do DC, as vacas do DP apresentaram maior porcentagem de prenhez que as do DC (40,0\% x 11,1\%; P<0,01), evidenciando melhores desempenhos reprodutivos devido à retirada do efeito negativo da lactação (Williams, 1990; Ferreira, 1992; Simeone \& Lobato, 1996). Isto possibilitou incremento nos ganhos médios diários (Figura 1), no peso vivo e na CC para as vacas do DP durante o acasalamento em relação ao grupo de vacas que continuaram amamentando até 26/03/99 (Tabela 1).

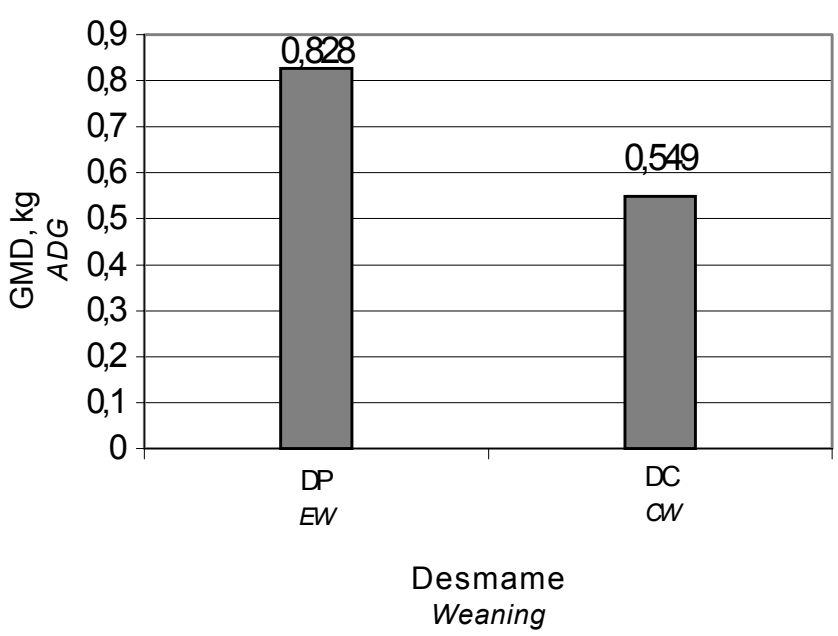

Figura 1 - Ganhos médios diários (GMD) da data do desmame precoce ao final do acasalamento, conforme o desmame, precoce (DP) ou convencional (DC).

Figure 1 - Average daily liveweight gain (ADG) from the early weaning date to the end of the breeding season, as the early $(E W)$ or the conventional weaning $(C W)$.

R. Bras. Zootec., v.31, n.3, p.1223-1229, 2002 
Tabela 2 - Taxa de prenhez (\%) das vacas nos anos de 1999 e 2000 , intervalos de partos (IEP) e número de animais $(\mathrm{N})$, segundo os tratamentos de desmame precoce (DP) ou convencional (DC)

Table 2 - Cows pregnancy rates (\%) in 1999 and 2000, calving interval (Cl) and number of animals (N), according to the early (EW) or the conventional weaning (CW)

\begin{tabular}{lccccc}
\hline $\begin{array}{l}\text { Tratamento } \\
\text { Treatment }\end{array}$ & $\begin{array}{c}\text { Prenhez 1999 } \\
\text { Pregnancy rate 1999 }\end{array}$ & $\mathrm{N}$ & $\begin{array}{c}\text { Prenhez 2000 } \\
\text { Pregnancy rate 2000 }\end{array}$ & $\mathrm{N}$ & $\begin{array}{c}\text { IEP } \\
C I\end{array}$ \\
\hline $\begin{array}{l}\text { Dias } \\
\text { Days }\end{array}$ & & & & & \\
DP & $40,0^{\mathrm{a}}$ & 50 & $51,4^{\mathrm{a}}$ & 27 & $399,8^{\mathrm{a}}$ \\
$E W$ & $11,1^{\mathrm{b}}$ & 43 & $20,0^{\mathrm{a}}$ & 08 & $403,8^{\mathrm{a}}$ \\
DC & & & & & \\
$C W$ & & &
\end{tabular}

Médias seguidas da mesma letra, na coluna, não diferem estatisticamente $(P>0,05)$.

Means followed by the same letter, in a column, do not significantly differ $(P>.05)$.

Apesar da maior taxa de prenhez das vacas submetidas ao DP, estes índices reprodutivos foram extremamente baixos para ambos os tratamentos, o que pode ser explicado pela baixa CC com que os animais iniciaram o acasalamento, não havendo tempo suficiente de recuperarem todo o estado corporal necessário à obtenção de maiores índices de prenhez. Sampedro (1993), na província de Corrientes, Argentina, para vacas cujos bezerros foram desmamados com três a três e meio meses de idade, obteve índices de prenhez de $93 \%$. Lobato et al. (2000) também encontraram taxas de prenhez superiores, quando vacas em pastagens melhoradas de inverno/primavera foram submetidas ao desmame aos 70 dias (DP) ou aos 176 dias (DC) de idade dos bezerros, $100 \%$ e $89,47 \%$, respectivamente. Os baixos índices de prenhez encontrados no presente trabalho podem ser explicados além da insuficiente $\mathrm{CC}$ e pesos vivos que as vacas apresentavam em 05/01/99 (35 dias após o início do acasalamento), pela pouca disponibilidade de matéria seca do campo nativo (730,5 kg matéria seca/ha) em 29/01/99.

Dados semelhantes foram obtidos por Gottschall \& Lobato (1996) trabalhando com vacas primíparas Nelore $\mathrm{x}$ Devon em campo nativo, as quais apresentavam $C C=2,1$ e 2,5 no início e no final do acasalamento, respectivamente. A taxa média de prenhez foi de apenas $7,9 \%$, mostrando que vacas adultas e primíparas com insuficiente $\mathrm{CC}$ no início da estação de monta apresentam baixos índices reprodutivos, como os observados neste experimento.

No ano de 2000, foi analisado somente o comportamento reprodutivo das vacas que ficaram prenhes em 1999 (Tabela 2).

As vacas dos tratamentos originais, DP e DC, apresentaram pesos vivos e CC de 444,7 kg e 2,71; $436,1 \mathrm{~kg}$ e 2,81, respectivamente, em 06/12/99, evidenciando suas semelhanças em peso vivo e CC no período que antecedeu a estação de monta de 1999/2000.

As médias do IEP das vacas do desmame precoce e convencional estão apresentadas na Tabela 2. Resultados da análise de sobrevivência indicaram diferenças não significativas nas curvas de sobrevivência para IEP das vacas dos dois tratamentos $(\mathrm{P}>0,05)$ pelo teste de Wilcoxon (SAS, 1996). Esta medida de performance reprodutiva pode ter sido afetada pela data de início do segundo acasalamento, uma vez que vacas podem ciclar antes da época de monta estabelecida. No entanto, os valores aqui determinados estão distantes de um IEP de +/- 365 dias, o qual é considerado ideal para que uma vaca obtenha uma cria por ano (Sacco et al., 1990). Sexo do bezerro e grupo genético da vaca também não influenciaram significativamente o IEP $(\mathrm{P}>0,05)$.

Laflamme \& Connor (1992) afirmaram que os intervalos reprodutivos são intimamente relacionados ao estado fisiológico das vacas ao início da estação de monta. Os resultados expressos na Tabela 1 mostram baixos peso vivo e CC. De acordo com Scaglia (1997), pesos e CC inadequados durante o acasalamento ocasionam reduzidos índices de prenhez, além de aumentarem o IEP (Osoro \& Wright, 1992).

Trabalho recente de Lobato et al. (2000), conduzido com primíparas mestiças aos três anos de idade com pastejo em pastagem melhorada durante o pré e pós-parto (no período inverno/primavera), evidenciou o quanto é desgastante para as vacas a lactação e seus efeitos nos índices reprodutivos. Tendo submetido as vacas a tratamentos de DP de seus 
bezerros aos $83,4 \mathrm{~kg}$ e 70 dias de idade em média, e de DC com 153,3 kg aos 176 dias de idade em média, determinaram $100 \%$ e $89,47 \%$ de prenhez, com IEP de DC 359,6 381,6 dias, respectivamente. Isto demonstra que mesmo em pastagens melhoradas, com elevado índice de repetição de prenhez $(89,47 \%)$, o IEP do tratamento DC teve um atraso de 16,6 dias em relação ao ideal de 365 dias.

Estes resultados demonstram que, em vacas sem a devida CC ao parto e ao início do acasalamento, decisões de manejo devem ser tomadas para obtenção de melhores índices reprodutivos: o percentual de prenhez e o IEP.

\section{Conclusões}

Vacas submetidas ao desmame precoce são mais pesadas, possuem melhores condições corporais ao final do acasalamento e apresentam maiores ganhos médios diários durante o acasalamento, proporcionando maior porcentagem de prenhez no ano da realização do desmame.

Melhores condições corporais durante o período reprodutivo refletem-se em maiores índices de prenhez.

Baixos escores de condição corporal inibem o efeito do desmame precoce sobre o intervalo de partos.

\section{Literatura Citada}

ASSOCIATION OF OFICIAL ANALYTICAL CHEMIST AOAC. Official methods of analysis. 12 ed. Washington, DC.: 1975. 1094 p.

Bergamaschi, H.; GUADAGNiN, M.R. Agroclima da Estação Experimental Agronômica. Porto Alegre: Universidade Federal do Rio Grande do Sul, 1990. 96p.

BOLDRINI, I.I. Dinâmica de vegetação de uma pastagem natural sob diferentes níveis de oferta de forragem e tipos de solo, Depressão Central, RS. Porto Alegre: Universidade Federal do Rio Grande do Sul, 1993. 262p. Dissertação (Doutorado em Zootecnia) - Universidade Federal do Rio Grande do Sul, 1993.

BRASIL. Ministério da Agricultura. Departamento Nacional de Pesquisa Agropecuária. Divisão de Pesquisa Pedológica, Levantamento de reconhecimento dos Solos do estado do Rio Grande do Sul. Recife, 1973. 431p. (Boletim Técnico, 30).

FERREIRA, A.M. Efeito da amamentação na reprodução de vacas: uma revisão. Pesquisa Agropecuária Brasileira, v.27, n.1, p.27-39, 1992.

FERREIRA, A.M. Nutrição e atividade ovariana em bovinos: uma revisão. Pesquisa Agropecuária Brasileira, v.28, n.9, p.1077-1093, 1993.
GOMEZ, K.A.; GOMEZ, A.A. Statistical procedures for agricultural research. 2.ed. New York: John Wiley, 1984. 680p.

GOTTSCHALL, C.S.; LOBATO, J.F.P. Comportamento reprodutivo de vacas de corte primíparas submetidas a três lotações em campo nativo. Revista Brasileira de Zootecnia, v.25, n.1, p.46-57, 1996.

HAYDOCK, K. P.; SHAW, N.H. The comparative yield method for estimating dry matter yield of pasture. Australian Journal of Experimental Agriculture and Animal Husbandry, v.15, n.76, p.663-670, 1975.

LAFLAMME, L.F.; CONNOR, M.L. Effect of postpartum nutrition and cow body condition at parturition on subsequent performance of beef cattle. Canadian Journal of Animal Science, v.72, n.4, p.843-851, 1992.

LOBATO, J.F.P.; BARCELLOS, J.O.J. Efeitos da utilização de pastagem melhorada no pós-parto e do desmame aos 100 ou 180 dias de idade no desempenho reprodutivo de vacas de corte. Revista Brasileira de Zootecnia, v.21, n.3, p.385-395, 1992.

LOBATO, J.F.P.; DERESZ, F.; LEBOUTE,E.M. et. al. Pastagens melhoradas e suplementação alimentar no comportamento reprodutivo de vacas de corte primíparas. Revista Brasileira de Zootecnia, v.27, n.1, p.47-53, 1998a.

LOBATO, J.F.P.; ZANOTTA Jr., R.L.D.; PEREIRA NETO, O.A. Efeitos das dietas pré e pós-parto na eficiência reprodutiva de vacas primíparas de corte. Revista Brasileira de Zootecnia, v.27, n.1, p. 857-862, 1998b.

LOBATO, J.F.P.; MÜLLER, A. ; PEREIRA NETO, O.A. et al. Efeitos da idade à desmama dos bezerros sobre o desempenho reprodutivo de vacas de corte primíparas. Revista Brasileira de Zootecnia, v.29, n.6, p.2013-2018, 2000.

LOWMAN, B.G.; SCOTT, N.; SOMERVILLE, S. Condition scoring beef cattle. Edinburgh: East of Scotland College of Agriculture, 1973. (Bulletin, 6).

OSORO, K.; WRIGHT, I.A. The effect of body condition, live weight, breed, age, calf performance, and calving date on reproductive performance of spring-calving beef cows. Journal of Animal Science, v.70, n.6, p.1661-1666, 1992.

PIMENTEL, C.A.; DESCHAMPS, J.C.; OLIVEIRA, J.A.F. et al. Effects of early weaning on reproductive efficiency in beef cows. Theriogenology, v.11, n.6, p.421-427, 1979.

QUADROS, S.A.F.; LOBATO,J.F.P. Efeitos da lotação no comportamento reprodutivo de vacas de corte primíparas. Revista Brasileira de Zootecnia, v.25, n.1, p.22-34, 1996.

ROVIRA, J.M. Manejo nutritivo de los rodeos de cría en pastoreo. Montevideo: Hemisferio Sur, 1996. 288p.

SÁ, I.G.; BARCELLOS, J.O.J.; LOBATO, J.F.P. et al. Efeito do desmame precoce sobre a variação de peso de vacas e bezerros de corte em três sub-épocas de parição. In: REUNIÃO DA SOCIEDADE BRASILEIRA DE ZOOTECNIA, 34., 1997, Juiz de Fora. Anais... Juiz de Fora: Sociedade Brasileira de Zootecnia, 1997. p.461.

SACCO, R.E.; BAKER,J.F.; CARTWRIGHT, T.C. et al. Measurements at calving for straightbred and crossbred cows of diverse types. Journal of Animal Science, v.68, n.10, p.3103-3108, 1990. 
SAMPEDRO, D. Efecto del destete precoz sobre la tasa de preñez y la ganancia de peso de los terneros. In: JORNADA DE DIFUSIÓN TÉCNICA. DESTETE PRECOZ EN CRÍA VACUNA, 1993, Concepción del Uruguay. Resumos... Entre Rios: INTA, 1993. 59p.

SAS INSTITUTE. SAS/STAT. User's Guide. 11.ed. Cary: 1996.

SCAGLIA, G. Nutricion y reproduccion de la vaca de cria: uso de la condición corporal. Montevideo: INIA, 1997. 15p. (Serie Tecnica, 91).

SIMEONE, A.; LOBATO, J.F.P. Efeitos da lotação animal em campo nativo e do controle da amamentação no comportamento reprodutivo de vacas de corte primíparas. Revista Brasileira de Zootecnia, v.25, n.6, p.1216-1227, 1996.

WILLIAMS, G.L. Suckling as a regulator of postpartum rebreeding in cattle: a review. Journal of Animal Science, v.68, n.3, p.831-852, 1990.
WILTBANK, J.N.; ROWDEN,W.W.; INGALLS, J.E. et al. Effect of energy level on reproductive phenomena on mature Hereford cows. Journal of Animal Science, v.21, n.2, p.219-225, 1962.

WILTBANK, J.N. Research needs in beef cattle reproduction. Journal of Animal Science, v.31, n.4, p.755-762, 1970.

Recebido em: 23/07/01

Aceito em: 02/04/02 